\title{
Evolution of the low-frequency pulse profile of PSR B2217+47
}

\author{
D. Michilli, ${ }^{1,2}$ J. W. T. Hessels,,${ }^{1,2}$ J. Y. Donner, ${ }^{3}$ J.-M. Grießmeier, ${ }^{4,5}$ \\ M. Serylak, ${ }^{6,7}$ B. Shaw ${ }^{8}$ B. W. Stappers,${ }^{8}$ J. P. W. Verbiest,${ }^{3,9}$
}

A. T. Deller,${ }^{10}$ L. N. Driessen, ${ }^{1,8}$ D. R. Stinebring, ${ }^{11}$ L. Bondonneau, ${ }^{4}$ M. Geyer, ${ }^{12}$ M. Hoeft,${ }^{13}$ A. Karastergiou, ${ }^{12,6,14}$ M. Kramer,,${ }^{9,8}$ S. Osłowski, ${ }^{10}$ M. Pilia, ${ }^{15,2}$ S. Sanidas ${ }^{1}$ and P. Weltevrede ${ }^{8}$

${ }^{1}$ Anton Pannekoek Institute for Astronomy, University of Amsterdam, Science Park 904, 1098 XH Amsterdam, The Netherlands

${ }^{2}$ ASTRON, the Netherlands Institute for Radio Astronomy, Postbus 2, 7990 AA, Dwingeloo, The Netherlands

${ }^{3}$ Fakultät für Physik, Universität Bielefeld, Postfach 100131, 33501 Bielefeld, Germany

${ }^{4}$ LPC2E - Université d'Orléans / CNRS, 45071 Orléans cedex 2, France

${ }^{5}$ Station de Radioastronomie de Nançay, Observatoire de Paris, PSL Research University, CNRS, Univ. Orléans, OSUC, 18330 Nançay, France

${ }^{6}$ Department of Physics \& Astronomy, University of the Western Cape, Private Bag X17, Bellville 7535, South Africa

${ }^{7}$ SKA South Africa, 3rd Floor, The Park, Park Road, Pinelands, 7405, South Africa

${ }^{8}$ Jodrell Bank Centre for Astrophysics, School of Physics and Astronomy, The University of Manchester, Manchester M13 9PL, UK

${ }^{9}$ Max-Planck-Institut für Radioastronomie, Auf dem Hügel 69, 53121 Bonn, Germany

${ }^{10}$ Centre for Astrophysics and Supercomputing, Swinburne University of Technology, P.O. Box 218, Hawthorn, VIC 3122, Australia

${ }^{11}$ Dept. of Physics and Astronomy, Oberlin College, 110 North Professor St., Oberlin, OH 44074, USA

${ }^{12}$ Oxford Astrophysics, Denys Wilkinson Building, Keble Road, Oxford OX1 3RH, UK

${ }^{13}$ Thüringer Landessternwarte Tautenburg, Sternwarte 7, 07778 Tautenburg, Germany

${ }^{14}$ Department of Physics and Electronics, Rhodes University, PO Box 94, Grahamstown 6140, South Africa

${ }^{15}$ INAF - Osservatorio Astronomico di Cagliari, via della Scienza 5, 09047 Selargius (Cagliari), Italy

email: danielemichilli@gmail.com

\begin{abstract}
An evolution of the low-frequency pulse profile of PSR B2217+47 is observed during a six-year observing campaign with the LOFAR telescope at $150 \mathrm{MHz}$. The evolution is manifested as a new component in the profile trailing the main peak. The leading part of the profile, including a newly-observed weak component, is steady during the campaign. The transient component is not visible in simultaneous observations at $1500 \mathrm{MHz}$ using the Lovell telescope, implying a chromatic effect. A variation in the dispersion measure of the source is detected in the same timespan. Precession of the pulsar and changes in the magnetosphere are investigated to explain the profile evolution. However, the listed properties favour a model based on turbulence in the interstellar medium (ISM). This interpretation is confirmed by a strong correlation between the intensity of the transient component and main peak in single pulses. Since PSR B2217+47 is the fourth brightest pulsar visible to LOFAR, we speculate that ISM-induced pulse profile evolution might be relatively common but subtle and that SKA-Low will detect many similar examples. In this scenario, similar studies of pulse profile evolution could be used in parallel with scintillation arcs to characterize the properties of the ISM.
\end{abstract}

Keywords. pulsars: individual: PSR B2217+47 - ISM: general - radio continuum: ISM 


\section{Introduction}

The pulse profile of a radio pulsar is obtained by folding the flux density measured over time at the pulsar spin period. Pulse profiles so obtained are generally stable over years (Helfand et al. 1975). This allows to correlate pulse profiles at different epochs in order to obtain precise times of arrival (TOAs) and thus to use pulsars as clocks in timing experiments (Edwards et al. 2006).

Exceptions exist to this scenario and different variations in pulse profiles are observed. Some are well understood (e.g. due to geodetic precession, Damour \& Ruffini 1974; Weisberg et al. 1989), while few others are still unpredictable. Examples of possible causes to the latter class are free precession (Cordes 1993; Stairs et al. 2000), changes in the pulsar magnetosphere (Lyne et al. 2010) and propagation effects in the interstellar medium (ISM, Backer et al. 2000; Lyne et al. 2001). Similarly to geodetic precession, free precession would cause an evolution of pulse profiles because of the change in the viewing angle. Changes in the pulsar magnetosphere could alter the radio emission (e.g. Kramer et al. 2006). The effect has been invoked by Lyne et al. (2010) when they found a correlation in the variability of pulse profile and spin-down rate of six pulsars; Karastergiou et al. (2011) also attribute another profile evolution to magnetospheric changes that Brook et al. (2014) relate to an encounter with an asteroid; Brook et al. (2016) developed a code to specifically look for correlations between profile and spin-down rate variations, finding 10 cases. Turbulence in the ISM could cause a transient evolution of the pulse profile if radio waves from the pulsar are deflected back to the line of sight (LoS). This could cause the appearance of an 'echo' of the main peak in the pulse profile. This effect has been previously reported only for PSR B0531+21 (Backer et al. 2000; Lyne et al. 2001) and in that case, the turbulence was connected with the Crab nebula surrounding the pulsar.

The first clear profile variation in PSR B2217+47 was reported by Suleymanova \& Shitov (1994). They attributed the effect to precession of the pulsar. It is important to note that Ahuja et al. (2005) detected a gradient in the dispersion measure (DM) of the pulsar of an unusually large intensity, indicating strong turbulence in the ISM along the pulsar's LoS. An observing campaign on PSR B2217+47 started in 2011 using the LOw-Frequency ARray (LOFAR, Stappers et al. 2011; van Haarlem et al. 2013) and the Lovell telescope. LOFAR observed the pulsar at $150 \mathrm{MHz}$ using both the telescope core and German, France and UK stations in stand-alone mode. Lovell observed the pulsar during the same time-span at $1500 \mathrm{MHz}$. Pulse profiles were obtained from each observation.

\section{Results}

The profile evolution of PSR B2217+47 is complex and continuous during the six-year observational campaign with the LOFAR telescope (Fig. 1a). Two different transient components can be identified in the trailing part of the profile evolving in phase, relative intensity and shape. The first one is apparent in the first observations and gradually shifts toward the main peak. It is brighter and single-peaked. The second one appears in 2014. It is weaker and formed by multiple peaks that evolve with different characteristics. It also shifts in phase but the low intensity and complex profile shape prevent us from precisely characterising its evolution. The leading part of the profile, including a newly-detected weak component (precursor), is stable throughout the campaign.

The pulse profile obtained with Lovell is also stable throughout the campaign. The spectral indices of the brighter and weaker transient components are $\alpha=-3.58 \pm 0.09$ 


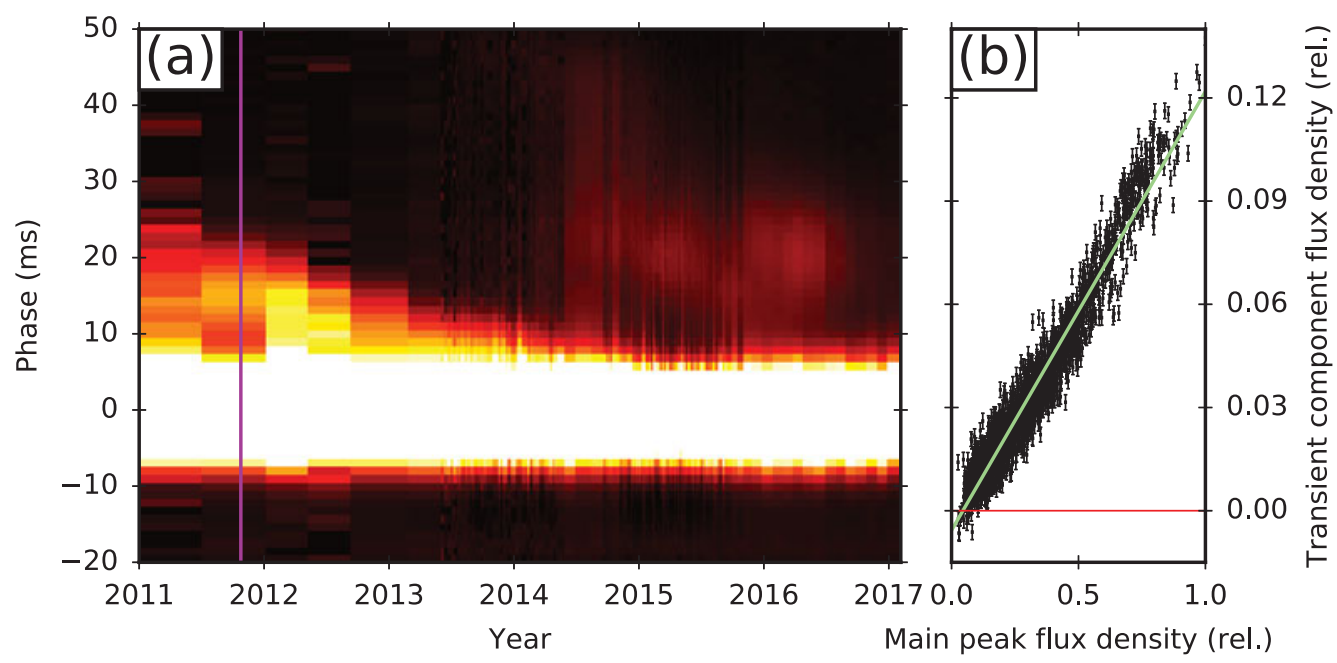

Figure 1. Panel a: evolution of the pulse profile detected by LOFAR during the six-year observational campaign. Profiles are normalized to unitary peak amplitude and aligned by crosscorrelating a standard template. The colour scale indicates the flux density, where black is the noise level and white is $15 \%$ of the peak value. A magenta line indicates the observation used in panel b. Panel b: scatter plot of the total flux density of the main peak and transient component for the single pulses recorded during a 15-minute observation. The green line represents a linear fit to the points.

and $-3.73 \pm 0.06$, respectively, consistent with a single value. Assuming a constant spectral index up to $1500 \mathrm{MHz}$, the steepness of the spectrum explains the non-detection of the transient components in Lovell observations.

We measured a new DM variation of the pulsar using LOFAR. The level of variation is an order of magnitude lower than that reported by Ahuja et al. (2005), however it is still significant and it is the only DM variation measured in a large sample of pulsars. Details and results of this DM study will be presented in a companion paper by Donner et al. (in prep.).

We selected one LOFAR observation where single pulses were stored and where the transient component was bright and well separated from the main peak. This observation is highlighted with a magenta line in Fig. 1a and was presented by Pilia et al. (2016). For every single pulse, we calculated the total flux density of the main peak and the transient component. The resulting scatter plot is presented in Fig. 1b. It shows that the flux density of the two components are highly correlated. This trend disappears when correlating the flux density of the main peak with the flux density of the transient component measured in a different rotation of the star.

\section{Discussion}

In a stellar precession scenario, DM variations are not expected and the profile will vary symmetrically on average. These two aspects of the profile evolution presented here would, then, be coincidental. Moreover, there is no physical reason for the large correlation found in the flux density of different components.

Since a self-consistent model of radio emission in pulsars is lacking, it is difficult to rule out magnetospheric effects. However, DM variations at the level detected in PSR B2217+47 have never been attributed to magnetospheric effects in the literature. Also, the asymmetric evolution of the profile in all the epochs is not readily explained 
within this scenario. Finally, a correlation between different components of the profile is normally not observed in pulsars.

Density variations in the ISM crossing the LoS explains the observed characteristics of the profile evolution. In this scenario, the transient components observed are all echoes of the main peak. The same structures could be responsible for the detected DM variations. Only the trailing part of the profile is affected due to the longer path travelled by the deflected radio waves. Since the transient component is a reflection of the main peak, the correlation between their flux densities is expected in this model. Finally, a quadratic evolution in the phase position of the transient components in time is expected within this model and this is consistent at least with the brighter transient component, though the low quality and paucity of early observations do not permit to put a strong constraint. A similar analysis for the weaker component is prevented by the low flux and complex profile.

PSR B2217+47 is the fourth brightest pulsar visible to LOFAR (Bilous et al. 2016). A similar profile variation would not be visible in weaker pulsars, using less sensitive telescopes or observing at higher frequencies. Therefore, we speculate that ISM-induced profile variations may be relatively common. With its large sensitivity at low frequencies, this hypothesis will be tested by the Square Kilometer Array (SKA). SKA-Low has the potential to discover many similar examples of ISM-induced profile variations. With a large sample, these phenomena could be used to study the ISM properties, offering a tool complementary to scintillation arcs (Stinebring et al. 2001).

\section{References}

Ahuja A. L., Gupta Y., Mitra D., Kembhavi A. K., 2005, MRAS 357, 1013

Backer D. C., Wong T., Valanju J., 2000, ApJ 543, 740

Bilous A. V., et al., 2016, A\&̈A 591, A134

Brook P. R., Karastergiou A., Buchner S., Roberts S. J., Keith M. J., Johnston S., \& Shannon R. M., 2014, ApJL 780, L31

Brook P. R., Karastergiou A., Johnston S., Kerr M., Shannon R. M., \& Roberts S. J., 2016, MRAS 456, 1374

Cordes J. M., 1993, in Phillips J. A., Thorsett S. E., \& Kulkarni S. R., eds, Astronomical Society of the Pacific Conference Series Vol. 36, Planets Around Pulsars. pp 43-60

Damour T. \& Ruffini R., 1974, Academie des Sciences Paris Comptes Rendus Serie Sciences Mathematiques 279, 971

Edwards R. T., Hobbs G. B., \& Manchester R. N., 2006, MRAS 372, 1549

Helfand D. J., Manchester R. N., \& Taylor J. H., 1975, ApJ 198, 661

Karastergiou A., Roberts S. J., Johnston S., Lee H., Weltevrede P., \& Kramer M., 2011, MRAS 415,251

Kramer M., Lyne A. G., O’Brien J. T., Jordan C. A., \& Lorimer D. R., 2006, Science 12, 549

Lyne A. G., Pritchard R. S., \& Graham-Smith F., 2001, MRAS 321, 67

Lyne A., Hobbs G., Kramer M., Stairs I., \& Stappers B., 2010, Science 329, 408

Pilia M., et al., 2016, A\&A 586, A92

Stairs I. H., Lyne A. G., \& Shemar S. L., 2000, Nature 406, 484

Stappers B. W., et al., 2011, A\& A 530, A80

Stinebring D. R., McLaughlin M. A., Cordes J. M., Becker K. M., Goodman J. E. E., Kramer M. A., Sheckard J. L., \& Smith C. T., 2001, ApJL 549, L97

Suleymanova S. A. \& Shitov Y. P., 1994, ApJL 422, L17

van Haarlem M. P., et al., 2013, A\&SA 556, A2

Weisberg J. M., Romani R. W., \& Taylor J. H., 1989, ApJ 347, 1030 